\title{
Red-Emitting Rhodamine Dyes for Fluorescence Microscopy and Nanoscopy
}

\author{
Kirill Kolmakov, Vladimir N. Belov,* Jakob Bierwagen, Christian Ringemann, \\ Veronika Müller, Christian Eggeling, and Stefan W. Hell*a]
}

\begin{abstract}
Fluorescent markers emitting in the red are extremely valuable in biological microscopy since they minimize cellular autofluorescence and increase flexibility in multicolor experiments. Novel rhodamine dyes excitable with $630 \mathrm{~nm}$ laser light and emitting at around $660 \mathrm{~nm}$ have been developed. The new rhodamines are very photostable and have high fluorescence quantum yields of up to $80 \%$, long excited state lifetimes of $3.4 \mathrm{~ns}$, and comparatively low intersystem-crossing rates. They perform very well both in conventional and in subdiffraction-resolution microscopy such as STED (stimulated emission depletion) and
\end{abstract}

GSDIM (ground-state depletion with individual molecular return), as well as in single-molecule-based experiments such as fluorescence correlation spectroscopy (FCS). Syntheses of lipophilic and hydrophilic derivatives starting from the same chromophore-containing scaffold are described. Introduction of two sulfo groups provides high solubility in water and a considerable rise in fluorescence quantum yield. The attachment of amino or thiol reactive

Keywords: dyes/pigments • fluorescence $\cdot$ fluorescent probes $\cdot$ isotopic labeling $\cdot$ microscopy groups allows the dyes to be used as fluorescent markers in biology. Dyes deuterated at certain positions have narrow and symmetrical molecular mass distribution patterns, and are proposed as new tags in MS or LC-MS for identification and quantification of various substance classes (e.g., amines and thiols) in complex mixtures. High-resolution GSDIM images and live-cell STED-FCS experiments on labeled microtubules and lipids prove the versatility of the novel probes for modern fluorescence microscopy and nanoscopy.

\section{Introduction}

Owing to their high photostabilities, high extinction, high fluorescent quantum yields, and low degree of triplet formation, rhodamine dyes are widely used as laser dyes and fluorescent markers for labeling proteins, nucleic acids, lipids, carbohydrates, toxins, hormones, and other biomolecules. ${ }^{[1]}$ Besides their use in biological imaging and single-moleculebased spectroscopy, rhodamines served well in the practical implementation of some new physical concepts, for example, those that helped to overcome the diffraction limit in (far-

[a] Dr. K. Kolmakov, Dr. V. N. Belov, Dipl.-Chem. J. Bierwagen, Dr. C. Ringemann, Dipl.-Phys. V. Müller, Dr. C. Eggeling, Prof. Dr. S. W. Hell

Department of NanoBiophotonics

Max Planck Institute for Biophysical Chemistry

Am Fassberg 11, 37077 Göttingen (Germany)

Fax: (+49) 5512012505

E-mail: vbelov@gwdg.de shell@gwdg.de

Supporting information for this article is available on the WWW under http://dx.doi.org/10.1002/chem.200902309. field) optical microscopy. "Nanoscopic" techniques ${ }^{[2]}$ such as STED (stimulated emission depletion) ${ }^{[2 \mathrm{a}, \mathrm{d}, \mathrm{h}]}$ PALM (photoactivation localization microscopy), ${ }^{[2 i, s]}$ STORM (stochastic reconstruction microscopy) ${ }^{[2 \mathrm{j}, \mathrm{t}-\mathrm{v}]}$ and GSDIM (ground-state depletion with individual molecular return) ${ }^{[3]}$ allowed the optical resolution to be improved from about $200-350 \mathrm{~nm}$ to 20-35 $\mathrm{nm}$ by switching between the dark and the bright states of a fluorescent marker. However, the rhodamine family lacks water-soluble compounds that combine high photostabilities and fluorescence quantum yields with absorbance and fluorescence band maxima lying in the far-red optical region.

Biological applications favor fluorescent dyes that absorb in the far-red or even near-infrared (IR) optical region. In contrast to visible and above all ultraviolet, excitation at wavelengths longer than $600 \mathrm{~nm}$ is mostly noninvasive and minimizes the unwanted background signal originating from cellular autofluorescence. The most convenient near-IR laser lines are between 630 and $650 \mathrm{~nm}$, as supplied by He$\mathrm{Ne}$, diode, or krypton ion lasers. A number of fluorescent dyes have been prepared to match these excitation sources. ${ }^{[4]}$ However, a survey of the patent literature revealed 
only one commercially available rhodamine dye, namely, Alexa 633, which was initially reported as a "sulfonated rhodamine derivative". ${ }^{[5]}$ Its exact structure was disclosed in 2007. ${ }^{[5]}$ It can be deduced that the photostability of Alexa 633 is similar to those of the cyanine dyes. ${ }^{[4]}$

Here we present novel rhodamine dyes, excitable at $630 \mathrm{~nm}$ and emitting at around $660 \mathrm{~nm}$. They are highly photostable, have fluorescence quantum yields of up to $80 \%$, relatively long fluorescence lifetime $(3.4 \mathrm{~ns})$, and low intersystem-crossing rates. The same chromophore-containing scaffold allows introduction of various functional groups and preparation of fluorescent dyes ranging from lipophilic to highly water soluble (hydrophilic), as well as reactive dyes for specific biological labeling. High-resolution GSDIM images of the labeled microtubular network in whole (fixed) cells and live-cell, single-molecule-based STED-FCS (fluorescence correlation spectroscopy) experiments with lipids illustrate the potential of the novel markers in modern fluorescence microscopy.

\section{Results and Discussion}

Background information on red fluorescent dyes: Fluorescence microscopy, nanoscopy, and FCS demand markers with "red" excitation and emission bands, high fluorescence quantum yields $\left(\Phi_{\mathrm{fl}}\right)$, high oscillator strengths (absorption coefficients), high photostability, low rates of formation of the "dark" triplet state, relatively long lifetimes of the excited states ( $>3 \mathrm{~ns}$ ), sufficient solubility in water, and a reactive group with a linker for conjugation with biological objects or other structures of interest. Moreover, the availability of lipophilic and hydrophilic derivatives of the same chromophore is desirable, as long as it provides additional flexibility in labeling substances of various polarities. Hydrophobicity would be advantageous for labeling polar substances such as lipid head groups, while lipophilic derivatives are useful for labeling nonpolar domains (e.g., lipid acyl chains).

Commercially available red-emitting dyes include the previously mentioned rhodamine Alexa 633, the cyanine dyes Alexa 647 and Cy 5, oxazines such as Evoblue 30, and the carbopyronine dyes Atto 647N and Atto 635, designed to match the preferred characteristics. Carbopyronine dyes are structurally similar to rhodamines and can serve as their competitive substitutes in the far-red spectral region. ${ }^{[6]}$ In particular, Atto $647 \mathrm{~N}$ has gained popularity as a dye for labeling in "nanoscopic" studies. Being very photostable, Atto $647 \mathrm{~N}$ has quite recently been applied for live-cell video-rate STED imaging. ${ }^{[7]}$ In the course of the study, the movements of synaptic vesicles inside the axons of cultured neurons were recorded with a spatial resolution of about $60 \mathrm{~nm}$. However, the low polarity of Atto $647 \mathrm{~N}$ is often disadvantageous. $^{[4]}$ The Atto 647N-labeled molecules may strongly stick to glass, for example, to microscope cover slides or to the walls of microcapillary injection tubes. As a consequence, Atto $647 \mathrm{~N}$ proves difficult for in vitro biologi- cal assays or for proper cellular injection. In addition, being lipophilic, Atto $647 \mathrm{~N}$ produces a considerable background in our immunostaining experiments largely due to its affinity for mitochondria. Moreover, when coupled to an antibody for immunostaining, Atto $647 \mathrm{~N}$ sometimes displays a strong increase in the intensity of an additional absorption peak at around $605 \mathrm{~nm}$ that gives no emission at all. The complex structure of Atto 647N and the other carbopyronine dyes precludes any further chemical modifications aimed at increased polarity and better solubility in water. On the other hand, the cyanine dyes Alexa 647 and Cy 5 and the rhodamine Alexa 633 are less photostable and have lower fluorescence quantum yields than Atto 647N. The following order of the fluorescence quantum yields and photoresistance was observed: Atto 633 $\geq$ Atto $647 \mathrm{~N}>$ Alexa 647 $>$ Alexa 633 $>$ Cy $5 .{ }^{[4]}$ Further, cyanine dyes have rather short fluorescence lifetimes of about $1 \mathrm{~ns}$, and we can rule them out as potential lead structures for further improvements. The short lifetimes of the excited states, presumably low fluorescence quantum yields, and moderate photostabilities of oxazine dyes such as Evoblue 30 (for structures, see ref. [4]) make them poor candidates for further optimization.

The commercially available dyes mentioned above are widely used nowadays in numerous microscopic and nanoscopic studies. Attempts to devise and improve photostable red-emitting dyes of other classes are being made in various research groups. Most recent publications on this topic describe water-soluble terrylenediimides, ${ }^{[8 a, b]}$ new hydrophilic BODIPY derivatives, ${ }^{[8 c]}$ and dicyanomethylene dihydrofurans. ${ }^{[8 \mathrm{~d}]}$ However, some important data on photophysical properties of the bioconjugates and microscopic applications of terrylene diimides and dicyanomethylene dihydrofurans ${ }^{[8 a, b, d]}$ are lacking. Therefore, here we consider and refer to commercially available dyes (mentioned above and in the Supporting Information), whose properties and performance are well described and are always possible to explore.

The path to novel red-emitting rhodamines: Having ruled out cyanines, oxazines, and carbopyronines as lead structures for the reasons mentioned above, we ultimately chose rhodamines as development candidates. Although quite a number of diverse rhodamines with a great variety of substituents have been explored, certain possibilities to shift the emission of rhodamine dyes to the red region still remain.

A very large bathochromic shift was reported for Rhodamine 700 with the skeleton of the well-known Rhodamine 101, in which the benzoic acid residue is replaced by a trifluoromethyl group. All rhodamines with a perfluoroalkyl group at the 9-position absorb and emit above $600 \mathrm{~nm} .^{[8 \mathrm{e}]}$ Unfortunately, the presence of a small and very strongly electron accepting group at C-9 of the xanthene fragment ("opposite" to the oxygen atom) makes this position very vulnerable to nucleophilic attack by water. As a result, such rhodamines decolorize rapidly in aqueous solution and they cannot be used as scaffolds.

On the other hand, Rhodamine 101 (Rh 101, for structure see refs. $[1 \mathrm{a}-\mathrm{c}, 10])$ is one of the most stable and brightest 
fluorophores known $\left(\Phi_{\mathrm{fl}}=\right.$ $\left.1^{[10]}\right)$. Due to the high degree of substitution and the planarity of the molecule, its carboxyl group is sterically hindered, and therefore coupling reactions with amines require drastic conditions or powerful activating agents. ${ }^{[1]}$ Importantly, amidation of $\mathrm{Rh} 101$ with secondary amines results in a redshift of the absorption and emission bands from 560 and $589 \mathrm{~nm}$ to 583 and $604 \mathrm{~nm}$, respectively, with no significant change in $\Phi_{\mathrm{fl}}{ }^{[12]}$

There are further tools for achieving the desired redshift. First, the excitation and emission bands are considerably shifted to the red if tetrafluorophthalic anhydride is used for rhodamine synthesis instead of phthalic anhydride. ${ }^{[13]}$ Second, a $\mathrm{C}=\mathrm{C}$ bond may be introduced into the six-membered ring, fused with the xanthene fragment in such a way that it conjugates with the adjacent benzene ring. By these means, the highest reported values of the adsorption and emission maxima (630 and $655 \mathrm{~nm}$, respectively) for a "regular" rhodamine were achieved in the rigidized xanthene derivative of structure 4 (see Scheme 1). ${ }^{[9]}$ However, 4 is unsuitable for bioconjugation. It is poorly soluble in water and lacks a convenient binding site. Moreover, its free carboxyl group may form colorless and nonfluorescent cyclic esters (lactones). This carboxyl group is sterically hindered, as in all rhodamines, and amidation reactions with primary amines (e.g., in proteins) would give primary amides that are known to form colorless and nonfluorescent cyclic spiroamides (due to addition of the $\mathrm{NH}$ group across the tetrasubstituted $\mathrm{C}^{9}=\mathrm{C}^{8 \mathrm{a} / \mathrm{bb}}$ bond in the central xanthene ring). ${ }^{[2 \mathrm{~m}, \mathrm{n}, \mathrm{o}]}$

Synthesis of tetrafluororhodamine 4 and its lipophilic and hydrophilic derivatives: We chose compound $\mathbf{4}$ as a scaffold for further derivatization. Synthesis of tetrafluororhodamine 4 (Scheme 1) started with $\mathbf{1}$, which was prepared from $m$ anisidine and acetone in the presence of dry ytterbium(III) triflate according to the known general method. ${ }^{[14]}$ Alkylation of 1 with 1-bromo-3-chloropropane was carried out similarly to the procedure described for its closest analogue, the corresponding 10-pivaloyl ester. ${ }^{[9 a]}$ Demethylation of tricyclic ether 2-Me was carried out under conditions reported for aromatic methyl esters. ${ }^{[15]}$ Conversion of the intermediate $\mathbf{2}-\mathrm{H}$ to rhodamine $\mathbf{4}$ was performed stepwise according to the known method for the synthesis of asymmetric xanthene derivatives. ${ }^{[9 a]}$ Condensation of $\mathbf{3}$ and $\mathbf{2}-\mathbf{H}$ in the presence of $\mathrm{POCl}_{3}$ affords the corresponding acid chloride as an intermediate. Aqueous workup leads to zwitterionic compound 4 , while quenching the reaction with methanol produces methyl ester $\mathbf{7}$, easily hydrolyzed to $\mathbf{4}$ under the proper conditions. Other esters of rhodamine $\mathbf{4}$ can be easily obtained in a similar way. In the long run, they may be interesting intermediates, because one of the fluorine atoms in the aromatic ring can be easily replaced with nucleophilic (secondary) aliphatic amines. ${ }^{[2]]}$ Such transformations may open a new route to diverse fluorescent dyes. With compound 4 as scaffold, attachment of the $N$-methyl- $\beta$-alanine bridge to the rhodamine core (see Scheme 1 for the synthetic sequence and structures) provided the crucial redshift (> $20 \mathrm{~nm}$ in a MeOH solution, see Table 1). Moreover, the sterically unhindered carboxyalkyl or carboxyl groups in compounds $\mathbf{5}$ are available for further smooth and high-yielding transformations. Note that in compound $\mathbf{4}$ the positions of the absorbance/emission maxima (616 and $641 \mathrm{~nm}$ in 
Table 1. Spectroscopic properties of the fluorescent dyes prepared in this study. ${ }^{[a]}$

\begin{tabular}{llllllll}
\hline $\begin{array}{l}\text { Com- } \\
\text { pound }\end{array}$ & $\begin{array}{l}\lambda_{\max } \\
(\mathrm{abs}) \\
{[\mathrm{nm}]}\end{array}$ & $\begin{array}{l}\lambda_{\max } \\
(\mathrm{fl} .) \\
{[\mathrm{nm}]}\end{array}$ & Solvent & $\begin{array}{l}\varepsilon \\
{\left[10^{-5} \mathrm{M}^{-1} \mathrm{~cm}^{-1}\right]}\end{array}$ & $\begin{array}{l}\Phi_{\mathrm{fl} .}[\%] \\
\text { in } \\
\mathrm{H}_{2} \mathrm{O}^{[\mathrm{b}]}\end{array}$ & $\begin{array}{l}\tau_{\mathrm{fl}} \\
{[\mathrm{ns}]}\end{array}$ & $\begin{array}{l}k_{\mathrm{ISCT}} \\
{\left[10^{-6} \mathrm{~s}^{-1}\right]^{[\mathrm{cc}]}}\end{array}$ \\
\hline $\mathbf{4}$ & 616 & 641 & $\mathrm{MeOH}$ & 63 & & \\
$\mathbf{7}$ & 632 & 655.5 & $\mathrm{MeOH}$ & & & & \\
$\mathbf{5 -} t \mathrm{Bu}$ & 638 & 661 & $\mathrm{MeOH}$ & 0.92 & 61 & & \\
$\mathbf{5 - M e}$ & 638 & 661.5 & $\mathrm{MeOH}$ & 0.66 & 62 & & \\
$\mathbf{5 - H}$ & 638 & 661.5 & $\mathrm{MeOH}$ & 0.73 & 53 & 3.6 & \\
$\mathbf{6}^{[\mathrm{d}]}$ & 637 & 660 & $\mathrm{H}_{2} \mathrm{O}$ & 0.94 & 80 & 3.4 & $2.5 / 0.3$ \\
$\mathbf{6 D}$ & 637 & 660 & $\mathrm{H}_{2} \mathrm{O}$ & 0.92 & 78 & 3.4 & $2.5 / 0.3$ \\
\hline
\end{tabular}

[a] $\lambda_{\max }$ (abs.), $\lambda_{\max }\left(\mathrm{fl}\right.$.), $\varepsilon, \Phi_{\mathrm{fl}}$, and $\tau_{\mathrm{fl}}$ are absorption maxima, emission maxima, extinction coefficient, fluorescence quantum yield, and lifetime, respectively. [b] Atto 633 was used as reference $\left(\Phi_{\mathrm{fl}}=64 \%\right.$ in aqueous buffer). [c] Intersystem crossing rate $k_{\mathrm{ISC}}$ and rate of $\mathrm{T}_{1} \rightarrow \mathrm{S}_{0}$ transition $k_{\mathrm{T}}$ measured by FCS under single-molecule conditions in water at $22{ }^{\circ} \mathrm{C} \cdot{ }^{[17]}$ [d] The fluorescence quantum yields of bioconjugates were $48 \%$ (for dye 6 and sheep anti-mouse antibodies) and $40 \%$ (for dye 6 and goat antirabbit antibodies).

$\mathrm{MeOH}$, respectively, see Table 1) are far from suitable. For attaching the bifunctional $\beta$-alanine "bridge" to the carboxyl group in $\mathbf{4}$ for providing the lipophilic fluorescent dyes 5-R, we used our previously developed technique. ${ }^{[2 \mathrm{p}]} \mathrm{We}$ established that utilization of $N$-methyl- $\beta$-alanine tert-butyl ester instead of the methyl ester has certain advantages. In contrast to $n$-alkyl esters, tert-butyl esters are easily cleaved under acidic conditions. Thus, in our synthesis of sulfonated water-soluble hydrophilic dye $\mathbf{6}$, the subsequent alkali-assisted saponification step becomes unnecessary. The absence of this step not only shortens the reaction sequence, but also prevents undesired fluorine substitution in the aromatic ring of dyes 5-R. (Under basic conditions in methanol one of the four fluorine atoms may be substituted by a strongly nucleophilic methoxide or hydroxide anion.) Another crucial step of the synthesis is introduction of the two sulfo groups. This is achieved by direct sulfonation, a straightforward procedure which may be considered to be a case of so-called allylic sulfonation of olefins. ${ }^{[2 r, 13]}$ Introduction of ester $5-t \mathrm{Bu}$ or acid 5- $\mathrm{H}$ into a large excess of $96-98 \% \mathrm{H}_{2} \mathrm{SO}_{4}$ at $0{ }^{\circ} \mathrm{C}$ followed by prolonged exposure at room temperature afforded the desired hydrophilic dye 6. Fortunately, these conditions (concentrated $\mathrm{H}_{2} \mathrm{SO}_{4}$ ) left the secondary amido group intact. Also importantly, the same starting material, tert-butyl ester 5- $t \mathrm{Bu}$, can be smoothly converted to the corresponding carboxylic acid $5-\mathrm{H}$ by simple treatment with $2 \mathrm{M} \mathrm{HCl}$ in 1,4-dioxane. In fact, the unsulfonated compound $\mathbf{5}-\mathrm{H}$ is the required lipophilic analogue of rhodamine 6. Later we managed to cleanly saponify methyl ester 5-Me to carboxylic acid 5-H using an excess of highly dilute aqueous $\mathrm{KOH}$ solution at room temperature. Nonetheless, the absence of methanol in the reaction mixture was important; with methanol, the saponification reaction invariably gave mixtures containing 5-H and a substance with very similar retention parameters (both on silica gel and the reversed phase), presumably the derivative whose aromatic fluorine atom was replaced by a methoxyl group. The solubility of $6-\mathrm{H}$ in water is high: up to $40-50 \mathrm{mg}$ in $1 \mathrm{~mL}$ of water at room temperature. The dark blue aqueous solutions produce intense red fluorescence when irradiated with incandescent or halogen lamps. Dissolution of $\mathbf{6}$ in methanol, aqueous methanol, or any methanol-containing mixtures is accompanied by noticeable esterification of the $\beta$-alanine fragment by methanol. Probably, the close proximity of the sulfonic acid residues to the carboxyl group assists nucleophilic addition (a case of an intramolecular acid catalysis). Unlike 6, lipophilic rhodamine $\mathbf{5}$ is very poorly soluble in water. On the other hand, the solubility of dye 5-H in DMF, THF, and even $\mathrm{CHCl}_{3}$ is very good. Due to the presence of the asymmetrically substituted secondary amide fragment, the molecules of compounds 5 and $\mathbf{6}$ (and their derivatives, see Scheme 1) have an $\mathrm{N}-\mathrm{C}$ chirality axis. The rotational barrier in asymmetrically substituted tertiary amides is high enough (ca. $18 \mathrm{kcal} \mathrm{mol}^{-1}$ ) that in the NMR spectrum two signals are observed for the $N$-methyl group (in ca. 1:2-1:3 ratio). The presence of the chirality axis makes the "opposite" methyl groups and the $\mathrm{CH}$ atoms attached to the "left" and "right" sides of the xanthene fragment nonequivalent (diastereotopic), and consequently in the NMR spectra of compounds 5 and $\mathbf{6}$ two additional sets of equally strong signals can be observed. For example, four signals of the methyl groups at the $\mathrm{C}=\mathrm{C}$ bond are clearly seen in ${ }^{1} \mathrm{H}$ NMR spectra of esters 5-Me and $\mathbf{5}-t \mathrm{Bu}^{[19]}$

Deuterated analogues: We also prepared the deuterated analogue of fluorescent dye $\mathbf{6}$ (compound 6D in Scheme 1, chemically identical to compound $\mathbf{6}$ ) for the following reasons. High-frequency $\mathrm{C}-\mathrm{H}$ stretching vibrations ( $v$ $\approx 3000 \mathrm{~cm}^{-1}$ ) considerably contribute to the nonradiative $\mathrm{S}_{1} \rightarrow \mathrm{S}_{0}$ transition characterized by the Frank-Condon factor $f_{0}$, which is associated with the maximum possible rate of nonemissive decay $\left(f_{0} \approx 1013 \mathrm{~s}^{-1}\right.$ in most chromophores with $\mathrm{C}-\mathrm{H}$ bonds). ${ }^{[16]}$ Therefore, isotopic substitution which creates $\mathrm{C}-\mathrm{D}$ bonds with considerably lower vibrational energy $\left(v \approx 2200 \mathrm{~cm}^{-1}\right)$ may reduce the rate of nonradiative deactivation, improve the fluorescence quantum yield, and prolong the excited-states lifetime. These effects are likely to be observed in rigid molecular systems (e. g., rhodamines) in which the $S_{1}$ and $S_{0}$ states are known to have very similar geometries, so that their potential-energy surfaces do not intersect. Consequently, the overall deactivation rate may be approximated by the internal conversion, which is determined by the Frank-Condon factor. Furthermore, deuterated and non-deuterated fluorophores with the same structure are expected to have the same (or nearly the same) intersystem-crossing rates $\left(S_{1} \rightarrow T_{1}\right)$. In view of all these factors, we hoped that the deuteration would increase the fluorescent quantum yields and excited-state lifetimes. Compound 6D was synthesized similarly to compound $\mathbf{6}$.

Only a very large excess of $\left[\mathrm{D}_{6}\right]$ acetone in the reaction with $m$-anisidine catalyzed by anhydrous ytterbium(III) triflate affords compound 1-Me with sufficiently high deuterium content. The structural fragment $\mathrm{C}\left(\mathrm{CD}_{3}\right)=\mathrm{CD}$ (the allyl protons) in compound $\mathbf{1 - M e}$ is especially prone to the $\mathrm{D} / \mathrm{H}$ 
exchange. For this reason, the subsequent alkylation with $\mathrm{Br}$ $\left(\mathrm{CH}_{2}\right)_{3} \mathrm{Cl}$ was performed in $\left[\mathrm{D}_{3}\right]$ acetonitrile in the presence of $\mathrm{Na}_{2} \mathrm{CO}_{3}$ (instead of $\mathrm{NaHCO}_{3}$ ). Cleavage of deuterated methyl ester 2-Me was performed with $48 \% \mathrm{DBr}$ in $\mathrm{D}_{2} \mathrm{O}$ mixed with AcOD. Other steps leading to compound 5$t \mathrm{Bu}$ did not involve reagents or solvents with easily exchangeable protons and were performed as for the undeuterated precursor. The reaction with $97 \% \mathrm{D}_{2} \mathrm{SO}_{4}$ in $\mathrm{D}_{2} \mathrm{O}$ (deuterated concentrated sulfuric acid), due to the fast exchange of the allyl protons, substantially improved the degree of deuteration in the fragment $\mathrm{C}$ $\left(\mathrm{CD}_{3}\right)=\mathrm{CD}$. As a result, we made up for the undesired exchange to hydrogen, and target compound 6D had satisfactory isotopic purity and a very narrow molecular mass distribution in its mass spectrum with two major peaks corresponding to the presence of 17 and $18 \mathrm{D}$ atoms (of 18 possible).

Reactive derivatives: To demonstrate the potential and performance of the new dyes as fluorescent markers for bioconjugation and microscopy it was necessary first to prepare corresponding reactive deriva-

tives (starting from compounds 6/6D and their lipophilic counterpart 5-H for comparison). Usually, amino- or thiolreactive derivatives of fluorescent dyes are used for labeling of proteins. For conjugation with amines, the free carboxyl group must be activated by formation of $N$-hydroxy(sulfo)succinimidyl $(\mathrm{NH}(\mathrm{S}) \mathrm{S})$ esters, phenyl esters with electron-acceptor groups in the benzene ring, or similar compounds with good leaving groups. The corresponding NHS ester of lipophilic rhodamine 5 -H proved to be disappointingly unstable, and tetrafluorophenyl ester $\mathbf{8}$ was prepared instead (Scheme 2). Ester $\mathbf{8}$ partly decomposed in the course of chromatographic isolation on silica gel. Therefore, the reaction mixture containing the active ester $\mathbf{8}$ was used for labeling D-erythro-sphingosine phosphocholine (lyso-SM, Scheme 2). Low chemical stability of ester $\mathbf{8}$ and the corresponding NHS derivative may be explained by the presence of the nucleophilic hydroxide group as counterion. The neg- atively charged $\mathrm{OH}^{-}$ion is invariably formed in the first step of any esterification of the carboxyl group in 5-H. If it could be exchanged quickly and under mild conditions with another, less nucleophilic anion (e.g., perchlorate) before workup of the reaction mixture, then the chances to isolate the final ester $\mathbf{8}$ (or the corresponding NHS ester) in a pure state would have been better. The NHS esters of hydrophilic rhodamines 6 and 6D, compounds 9 and $9 D$, turned out to be comparatively stable. They were isolated by reversedphase preparative HPLC with gradient elution with aqueous acetonitrile containing $0.1 \%(\mathrm{v} / \mathrm{v})$ of TFA, lyophilized, and stored at $-20^{\circ} \mathrm{C}$ under argon. Under these conditions, the content of the corresponding acids 6/6D (the hydrolysis products) in these dark blue solids increased from 3-5\% up to $7-10 \%$ over two months. The higher stability of the NHS esters $\mathbf{9}$ and $\mathbf{1 1}$ may be explained by the absence of any nucleophilic counterion (the compounds themselves exist in 
the form of zwitterions, with sulfonate as anion). In the reaction with lyso-SM, active ester 9 afforded $\mathbf{1 2}-\mathrm{SO}_{3} \mathrm{H}$ (Scheme 2). Maleimide derivative $\mathbf{1 1}$ was prepared directly from 9 and 10 by combining them in DMF with $\mathrm{Et}_{3} \mathrm{~N}$ as base. It was also isolated by HPLC. The maleimide ring in $\mathbf{1 1}$ proved to be sensitive towards methanol even at room temperature (e.g., on dissolving the sample in methanol during preparation of the mass-spectroscopic sample).

Photophysical properties of the new rhodamine derivatives: Spectroscopic properties of fluorescent dyes 4-7 are given in Table 1. Lipophilic compounds $\mathbf{4}, \mathbf{5}$, and $\mathbf{7}$ were studied in methanolic solutions, and hydrophilic compounds 6/6D in water. All the dyes form solutions of vibrant blue color with intense red fluorescence, best visible on illumination with an incandescent lamp. As can be seen in the table, the required absorption and emission parameters of the lipophilic $(5-\mathrm{H})$ and hydrophilic (6 and 6D) fluorescent dyes have been achieved. The absorption and emission maxima were found to be at 637 and $660 \mathrm{~nm}$, respectively, similar to Alexa 633 and Atto $647 \mathrm{~N}$. The Stokes shift $(23 \mathrm{~nm})$ is considerably larger that that of Alexa $633(15 \mathrm{~nm})$ and similar to that of Atto $647 \mathrm{~N}(25 \mathrm{~nm})$. All fluorescence quantum yields were obtained for aqueous solutions. These values are considerably higher for the hydrophilic (sulfonated) dyes 6/6D (78$80 \%)$ than for the lipophilic substances: Atto $647 \mathrm{~N}$ (64\%) or rhodamines 4, 5, and 7 (53-63\%). Furthermore, in water dyes 6/6D (or 5- $\mathrm{H}$ in $\mathrm{MeOH}$ ) have relatively long fluorescence lifetimes of $3.4 \mathrm{~ns}$, similar to that of Atto $647 \mathrm{~N}$ (3.4 ns) and much larger than those of cyanine dyes (ca. $1 \mathrm{~ns})$. The rates of intersystem crossing $k_{\mathrm{ISC}}=2.5 \times 10^{6} \mathrm{~s}^{-1}$ and triplet depopulation $k_{\mathrm{T}}=3 \times 10^{5} \mathrm{~s}^{-1}$ of both dyes 6/6D are common to rhodamines, ${ }^{[17]}$ and under STED conditions they are as photostable as Atto $647 \mathrm{~N} .{ }^{[18]}$ Isotopic substitution did not affect the spectroscopic properties of the dye. Comparison of the photophysical characteristics of $\mathbf{6}$ with those of the deuterated analog $6 \mathrm{D}$ revealed no changes in fluorescence quantum yield, lifetime, or crossing rates to the triplet state.

Cellular labeling: The sulfo groups in rhodamines 6/6D, apart from providing excellent solubility in water, prevent aggregation of the dye molecules in water or aqueous buffers. This is the most probable explanation for the drastic rise in the fluorescence quantum yield that we observed (from $53 \%$ for the lipophilic compound 5- $\mathrm{H}[\mathrm{MeOH}]$ up to $80 \%$ for the hydrophilic derivative $\left.6\left[\mathrm{H}_{2} \mathrm{O}\right]\right)$. As a result, these dyes are superior in many respects to Atto 647N. The polar hydrophilic dyes 6/6D are not integrated into biological membranes and can be used at high concentrations in labeling experiments. ${ }^{[18]}$ The degree of labeling achieved with antibodies can be controlled by dosage of active NHS esters 9/9D. Typically anti-rabbit and anti-mouse antibodies were labeled to a degree of 2.5 to 6 . The fluorescence quantum yields of the conjugates were found to be $48 \%$ (for dye 6 and sheep anti-mouse antibodies) and $40 \%$ (for the dye 6 and goat anti-rabbit antibodies). Decrease in fluorescence quantum yields after conjugation with antibodies is normal and well-described. ${ }^{[2 \mathrm{p}]}$

Mass spectra of the deuterated analogues: Compound 6D, the deuterated analogue of dye $6\left(\mathrm{C}_{42} \mathrm{H}_{41} \mathrm{~F}_{4} \mathrm{~N}_{3} \mathrm{O}_{10} \mathrm{~S}_{2}, M=\right.$ $887)$, has up to 18 hydrogen atoms replaced with deuterium at certain aliphatic groups and at $\mathrm{C}=\mathrm{C}$ bonds (Schemes 1 and 2). As a result, in the negative-mode ESI mass spectrum the highest peak with $m / z 904[M-\mathrm{H}]$ corresponded to the molecular formula $\mathrm{C}_{42} \mathrm{H}_{23} \mathrm{D}_{18} \mathrm{~F}_{4} \mathrm{~N}_{3} \mathrm{O}_{10} \mathrm{~S}_{2}(M=905)$. The presence of 18 deuterium atoms indicates the maximal possible degree of substitution with deuterated acetone as precursor. However, peaks with lower masses are present, too. Molecular mass distribution (negative mode) of the molecular ion peaks in compound 6D was the following: $\mathrm{m} / \mathrm{z}$ (\%) 900 (15), 901 (36), 902 (67), 903 (94), 904 (100), 905 (46), 906 (18). After subtraction of the natural deuterium content, this molecular mass distribution corresponds to the following numbers of new deuterium atoms introduced in the course of the labeling procedures: $901\left(\mathrm{D}_{14}\right): 8 \% ; 902\left(\mathrm{D}_{15}\right): 13 \% ; 903$ $\left(D_{16}\right): 24 \%$; $904\left(D_{17}\right): 43 \%$; $905\left(D_{18}\right): 5 \% .{ }^{[19]}$ Molecular mass distribution patterns in compounds 6D and 9D were found to be identical, and no stable isotope "leakage" was observed in the course of the chemical transformations. Similar molecular mass distribution patterns can be easily found in the positive-mode ESI mass spectra of the sulfonated rhodamines 6D and 9D, too. However, in this mode these spectra are complicated by clustering with sodium and other positively charged ions. Compounds 6D and 9D have the same narrow molecular mass distribution patterns. Therefore, all their derivatives can be easily recognized and identified by mass spectra. Their characteristic feature is the symmetry of the molecular ion peaks due to the presence of the ions with lower molecular masses. ${ }^{[19]}$ Moreover, the selective derivatization of amines (with compound 9D) or thiols (with the deuterated analogue of the maleimide 11) may enable group identification in biologically important substance classes simply by comparison of the ESI mass spectra in the native sample before and after the reaction with the pairs of reagents $\mathbf{9}$ and $\mathbf{9 D}$ or $\mathbf{1 1}$ and its deuterated analogue. Importantly, the deuterated reagents (e.g., 9D) produce not only a new molecular mass distribution pattern in the derivatives, but also develop a considerable isotopic shift of 17-18 Da. Therefore, the new peaks do not overlap with the peaks of the derivatives obtained from compounds 9 or 11. It may even be advantageous to use $\mathbf{9}$ and $9 D$ (or $\mathbf{1 1}$ and its deuterated analogue) as mixtures for derivatization of proteins, tryptic peptides, and other complex biological samples. For very complex mixtures, HPLC separation prior to mass spectroscopic analysis may be used. We established that under reversed-phase HPLC conditions compounds 6 and 6D, as well as 9 and 9D, are inseparable and have the same retention times. Thus, their derivatives are also expected to give the same peaks under HPLC conditions. An important feature is the lower peak intensity of the molecular ions of the deuterated substances (6D and 9D) compared with the undeuterated analogues (6 and 9) at the same con- 
centrations. Thus, the relative content of a deuterated compound in a reagent mixture with an unlabeled counterpart must be 3-4 times higher, so that the intensities of the two peaks in the mass spectrum of the derivatized analyte will be comparable and easy to find. New molecular mass distribution tags introduced here are colored and highly fluorescent. These properties are indispensable for analytical purposes, because fluorescence and mass spectroscopy detection limits are similar (picograms of an analyte can easily be detected).

Far-field fluorescence nanoscopy: Compound 6 performs excellently in far-field fluorescence imaging and especially as a label in STED ${ }^{[18 b]}$ and GSDIM nanoscopy. ${ }^{[3]}$ The spatial resolution of standard far-field optical microscopy is limited by the diffraction of light to about $200 \mathrm{~nm}$ for visible light. ${ }^{[20]}$ Breaking of the diffraction barrier has been achieved by STED microscopy by transiently switching off the ability of a dye to emit everywhere but at isolated subdiffraction-sized spots. ${ }^{[2 a, d, h, 18]}$ In the STED method, switching off is realized by stimulated emission induced by an additional laser light. Effective fluorescence emission is constrained to a subdiffraction spot by overlaying the excitation focus with an intensity distribution for stimulated emission featuring a local zero. $^{[2 \mathrm{a}, \mathrm{d}, \mathrm{h}]}$ A high-resolution image is usually formed by scanning the overlapped foci over the sample and recording the fluorescence on a point detector. The final spatial resolution is determined by the intensity of the stimulated emission laser. The GSDIM method, ${ }^{[3]}$ on the other hand, relates to single-molecule techniques such as PALM (photo-activatable localization microscopy) ${ }^{[2 \mathrm{i}, \mathrm{s}]}$ or STORM (stochastical optical reconstruction microscopy), ${ }^{[2 \mathrm{j}, \mathrm{k}, \mathrm{t}-\mathrm{v}]}$ in which imaging of the stochastically switched-on single emitters is performed. Spatial position of the single isolated molecules is determined with subdiffraction precision from the signal detected with a camera. Plotting of the positions of repeatedly switched-on and -off molecules creates the final high-resolution image. Here, the spatial resolution depends on the number of photons detected from an isolated single molecule. In contrast to PALM and STORM, which apply special photoswitchable or photoactivatable labels, in the GSDIM method photoswitching is performed with conventional fluorophores by randomly shelving more than $90 \%$ of the molecules at a time into a dark state such as the triplet state. ${ }^{[3]}$ Over time, single molecules blink on and off. To achieve the desired switch-off level of greater than $90 \%$, the lifetime of the dark state must be long (milliseconds to seconds), which in the GSDIM approach is usually achieved by embedding the dyes in poly(vinyl alcohol), PVA, or using oxygen-consumption systems and exciting with a laser intensity greater than $10-100 \mathrm{~kW} \mathrm{~cm}^{-2}$. Recently, other approaches based on reducing and oxidizing agents were presented for oxazine and rhodamine dyes. ${ }^{[21]}$ Compound 6 combines the parameters most important for GSDIM: high quantum yield to detect a large number of photons per single-molecule event and optical shelving of $97 \%$ of all molecules into their dark (triplet) state (with an average lifetime of ca. $50 \mathrm{~ms}$ in PVA) following excitation with less than $10 \mathrm{~kW} \mathrm{~cm}^{-2}$. ${ }^{[19]}$ Consequently, GSDIM imaging of biological samples stained with compound 6 showed significantly enhanced resolution over conventional wide-field imaging. An illustrative example of a GSDIM image of a cellular $\beta$-tubulin network in the whole PtK2 cells immunolabeled with compound $\mathbf{6}$ is shown in Figure 1. Comparison with the conventional wide field

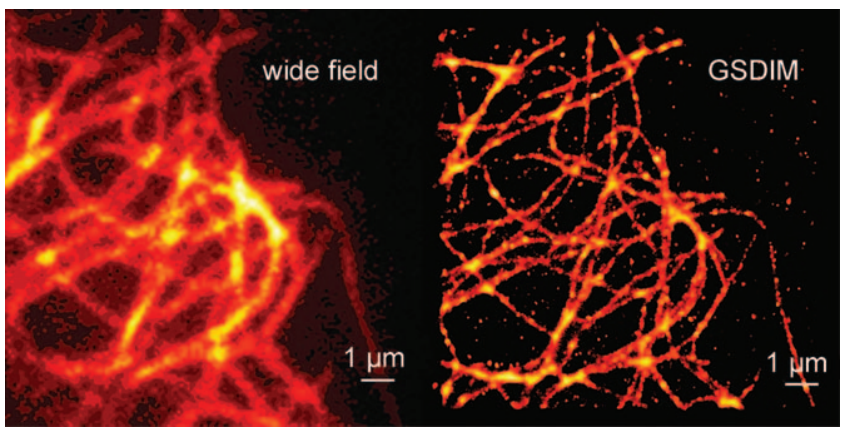

Figure 1. Conventional wide-field (left) and GSDIM (right) images of $\beta$ tubulin strands in whole fixed PtK2 cells stained with sheep anti-mouse secondary antibodies labeled with 6 (taken as NHS ester 9). The spatial resolution is approximately $300 \mathrm{~nm}$ in the wide-field image and $30 \mathrm{~nm}$ in the GSDIM image.

image indicates that the optical resolution is tenfold improved down to about $30 \mathrm{~nm}$. Features of the network hidden in the wide-field image can now clearly be resolved. Besides the protruding tubulin fibers, some randomly distributed fluorescent spots are detected in the GSDIM image. This is a well-known feature of nanoscopically resolved immunostained samples and is most likely explained by unspecific antibody binding. ${ }^{[2 \mathrm{~m}, \mathrm{o}, \mathrm{r}, 3]}$

Compound 6 performs much better in GSDIM than other red-emitting dyes such as Atto $647 \mathrm{~N}$ or Alexa 647 and Cy 5. ${ }^{[19]}$ Atto $647 \mathrm{~N}$ is unfavorable for GSDIM since its darkstate transition probability is too low $\left(k_{\mathrm{ISC}}<10^{6}\right)$, ${ }^{[17 b]}$ and consequently only up to $60 \%$ of all molecules can be shelved into the dark at a time. ${ }^{[3]}$ In contrast to the $97 \%$ of dark-state shelving for 6, up to 75 and $90 \%$ of Alexa 647 and Cy5 molecules, respectively, can be switched off at a time in PVA; the average dark-state lifetime is about $50 \mathrm{~ms}$ for 6 and only 20-25 ms for the mentioned cyanine dyes. ${ }^{[19]}$ Moreover, the laser intensity needed to attain this maximum switch-off ratio is less than $10 \mathrm{~kW} \mathrm{~cm}^{-2}$ for 6 but $40 \mathrm{~kW} \mathrm{~cm}^{-2}$ for Cy 5. Also, photobleaching is more pronounced for the cyanine dyes: After ten switch-on/-off cycles more than $20 \%$ of all Cy 5 or Alexa 647 molecules are photobleached, while almost $90 \%$ of $\mathbf{6}$ is still fluorescent. ${ }^{[19]}$ High photostability is favorable in the GSDIM protocol for reaching the highest possible contrast or for repetitive studies of dynamical events

STED-FCS: We performed STED-FCS experiments on sphingomyelin (SM) lipids labeled with hydrophilic compound 6 or the lipophilic compound 5-H (Scheme 2). Recent FCS studies on the dynamics of Atto647N-labeled 
SM in the plasma membrane of living PtK2 cells revealed that $\mathrm{SM}$ is transiently (ca. $10 \mathrm{~ms}$ ) trapped on small spatial scales $(<20-30 \mathrm{~nm})$ due to cholesterol-assisted complexation to other membrane constituents such as proteins. ${ }^{[22]}$ Trapping could, however, only be observed in spots smaller than $100 \mathrm{~nm}$ formed by STED; conventional confocal spots, about $250 \mathrm{~nm}$ in size, were too large to distinguish normal from heterogeneous SM diffusion. Figure 2 shows FCS data
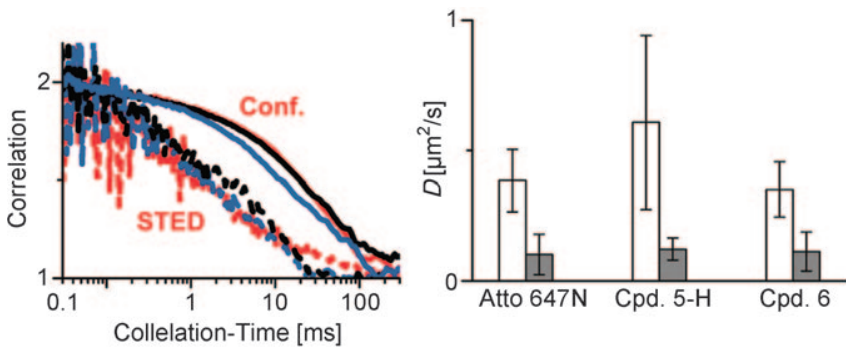

Figure 2. STED-FCS measurements on fluorescent SM lipid analogues in living Ptk2 cells: Correlation data of ATTO 647N- (black), 6- (red), and compound 5-H-labeled SM (blue) for confocal (solid lines "Conf.") and STED recording (dotted lines "STED"), that is, focal spots of about $240 \mathrm{~nm}$ and about $45 \mathrm{~nm}$ in diameter (left panel) and corresponding average diffusion constants $D$ calculated from the decay times of the correlation data from confocal (white bars) and STED (gray bars) recordings (right panel). The lower $D$ in the STED recordings renders heterogeneous diffusion of the lipids due to trapping. The extent of trapping (i.e., values of $D$ in STED) is the same regardless of the different polarities of the labels Atto 647N, 5-H ("Cpd. 5-H") or compound 6 ("Cpd. 6"). The error bars reveal the standard deviation from the analysis of more than 30 correlation curves recorded on different parts of the same cell and from different cells.

of fluorescent SM analogues labeled with Atto 647N, 6, or 5- $\mathrm{H}$ incorporated into the plasma membrane of living PtK2 cells for conventional confocal microscopy and high-resolution STED nanoscopy recordings. Diffusion of the fluorescent lipids through the focal spots with a diameter of about $240 \mathrm{~nm}$ (confocal) and about $45 \mathrm{~nm}$ (STED), which were placed on the plasma membrane, resulted in FCS correlation data dropping off with a decay time characteristic of the lipid focal transit time. For the confocal recordings the decay time of the correlation data recorded for 6-labeled SM is slightly shorter than for 5-H- and Atto647N-labeled SM. Labeling was performed by replacing the long native acyl chain at the second position of the SM molecule by a short acyl chain carrying the dye label, and thus compounds 12- $\mathrm{H}$ and 12- $\mathrm{SO}_{3} \mathrm{H}$ (Scheme 2) were synthesized starting from lyso-SM and active esters 8 and 9. In 12-H the whole lipophilic dye residue mimics the "second" long alkyl chain of sphingomyelin, while in $\mathbf{1 2}-\mathrm{SO}_{3} \mathrm{H}$ only one fatty acid chain is present. The higher polarity of $\mathbf{6}$ results in slightly less tight membrane anchoring of the corresponding SM analogue and thus in slightly faster diffusion, as was previously more distinctly revealed for the even more polar dye Atto 532 attached to lyso-SM. ${ }^{[22]}$ Due to the smaller focalspot diameter, the correlation data of the STED recordings are shifted to shorter times. However, this shift is less than that expected from the model based on free-diffusing species. The diffusion constants $D=d^{2} /\left(8 \ln 2 \tau_{\mathrm{D}}\right)$ calculated from the focal diameters $d$ and the decay times of the correlation curves are much smaller for the STED than for the confocal recordings (Figure 2) and render the heterogeneous diffusion of the SM lipids in the plasma membrane of living cells due to the mentioned nanoscale trapping: the decay of the STED-FCS data is dominated by trapping. Most strikingly, the STED-FCS data recorded for the SM analogues labeled with dyes of different polarities $(\mathbf{5}-\mathrm{H}$ is slightly more lipophilic than Atto $647 \mathrm{~N}$, and both are much more lipophilic than 6), show no significant differences. The trapping-dominated diffusion constants $D$ are the same for all three SM lipids. This negligible influence of the dye on the trapping parameters strongly supports the conclusion that the observed complexes are not caused or even influenced by the dye label. Trapping seems to be assisted by molecular groups of the lipid such as the hydrogen bonds of the sphingosine residue. Consequently, our observations are very likely to reflect the true behavior of the endogenous SM. ${ }^{[21]}$

\section{Conclusions}

New fluorescent dyes and markers for imaging and labeling in the red optical region were devised. They may be excited with irradiation at $633 / 635 \mathrm{~nm}$, emit light at around $660 \mathrm{~nm}$ with high fluorescence quantum yields in solution in the free state or when conjugated with biological substrates. The dyes are very photostable and have low intersystem-crossing rates and relatively long (3.4 ns) excited-state lifetimes. We prepared hydrophilic (soluble in water and aqueous buffers, 6/6D) and lipophilic (hydrophobic, insoluble in water or aqueous buffers, e.g., 5-H) forms of the dyes. This feature would have been much more difficult to achieve with the spectroscopically similar carbopyronines, because the preparation of hydrophilic carbopyronines is a tedious and multistep procedure. A carboxyl reactive site for conjugation with various functional groups (e.g., amines or thiols) is introduced by using tert-butyl 3-( $N$-methylamino)propionate as a bifunctional intermediate. If sulfonation follows, the saponification step is skipped. Compounds 6/6D perform excellently in far-field fluorescence nanoscopy techniques such as STED or GSDIM and are equally ideal for STED-FCS studies on diffusing lipids in the plasma membrane of living cells. We expect the novel dyes to be not only versatile labels for nanoscopy and single-molecule based studies but also helpful tools for multicolor experiments that can bring forth new insights into physical, chemical, or biological problems.

\section{Acknowledgements}

The authors are grateful to Donald Ouw for measuring the optical spectra, fluorescence quantum yields, and degrees of labeling. Excellent assistance of Dr. Ellen Rothermel, who performed the conjugation reactions, 
is acknowledged. We highly appreciate the assistance of Reinhard Machinek, Dr. Holm Frauendorf, and their co-workers, who recorded numerous NMR and mass spectra at the Institut für Organische und Biomolekulare Chemie (Georg-August-Universität Göttingen).

[1] For a general description and spectral range of rhodamines as fluorescent dyes, see: a) M. Beija, C. A.. Afonso, J. M. G. Martinho, Chem. Soc. Rev. 2009, 38, 2410-2433; M. S. T. Gonçalves, Chem. Rev. 2009, 109, 190-212; b) R. P. Haugland, A Guide to Fluorescent Probes and Labelling Technologies, Invitrogen, Carlsbad, 2005, pp. 11-37; c) for the use of rhodamines in bioconjugation techniques, see: G. T. Hermanson, Bioconjugate Techniques, Academic Press, Amsterdam, 2008, pp. 415-430; for laser dye properties of rhodamines, see: d) F. P. Schäfer in Dye Lasers (Ed.: F. P. Schäfer), Springer, Berlin, 1990, pp. 3-4; e) K.-H. Drexhage, Dye Lasers (Ed.: F. P. Schäfer), Springer, Berlin, 1990, p. 158; f) K.-H. Drexhage, Dye Lasers (Ed.: F. P. Schäfer), Springer, Berlin, 1990, pp. 175180; g) K.-H. Drexhage, Top. Appl. Phys. 1973, 1, 155-200.

[2] For reviews, see: a) S. W. Hell, Science 2007, 317, 1749-1753; b) M. Fernández-Suárez, A. Y. Ting, Nat. Rev. Mol. Cell Biol. 2008, 9 , 929-943; c) D. Evanko, Nat. Methods 2009, 6, 19-20; d) S. W. Hell, Nat. Methods 2009, 6, 24-32; e) X. Zhuang, Nat. Photonics 2009, 3, 365-367; f) J. Lippincott-Schwartz, S. Manley, Nat. Methods 2009, 6, 21-23; g) B. Huang, M. Bates, X. Zhuang, Annu. Rev. Biochem. 2009, 78, 993-1016; for recent reports, see: h) S. W. Hell, J. Wichmann, Opt. Lett. 1994, 19, 780-782; i) E. Betzig, G. H. Patterson, R. Sougrat, O. W. Lindwasser, S. Olenych, J. S. Bonifacino, M. W. Davidson, J. Lippincott-Schwartz, J. F. Hess, Science 2006, 313, 16421645; j) M. J. Rust, M. Bates, X. W. Zhuang, Nat. Methods 2006, 3, 793-795; k) M. Bates, B. Huang, G. T. Dempsey, X. Zhuang, Science 2007, 317, 1749-1753; 1) S. T. Hess, T. P. K. Girirajan, M. D. Mason, Biophys. J. 2006, 91, 4258-4272; m) J. Fölling, V. Belov, R. Kunetsky, R. Medda, A. Schönle, A. Egner, C. Eggeling, M. Bossi, S. W. Hell, Angew. Chem. 2007, 119, 6382-6386; Angew. Chem. Int. Ed. 2007, 46, 6266-6270; n) J. Fölling, V. Belov, D. Riedel, A. Schönle, A. Egner, C. Eggeling, M. Bossi, S. W. Hell, ChemPhysChem 2008, 9, 321-326; o) M. Bossi, J. Fölling, V. N. Belov, V. P. Boyarskiy, R. Medda, A. Egner, C. Eggeling, A. Schönle, S. W. Hell, Nano Lett. 2008, 8, 2463-2468; p) V. P. Boyarskiy, V. N. Belov, R. Medda, B. Hein, M. Bossi, S. W. Hell, Chem. Eur. J. 2008, 14, 1784-1792; q) S. M. Polyakova, V. N. Belov, C. Eggeling, S. F. Yan, C. Ringemann, G. Schwarzmann, A. de Meijere, S. W. Hell, Eur. J. Org. Chem. 2009, 5162-5177; r) V. N. Belov, M. L. Bossi, J. Fölling, V. P. Boyarskiy, S. W. Hell, Chem. Eur. J. 2009, 15, 10762-10776; s) H. Schroff, C. G. Galbraith, H. White, J. Gilette, S. Olenych, M. W. Davidson, E. Betzig, Proc. Natl. Acad. Sci. USA 2007, 104, 2030820313; t) M. Bates, B. Huang, G. T. Depsey, X. Zhuang, Science 2007, 317, 1749-1753; u) B. Huang, W. Wang, M. Bates, X. Zhuang, Science 2008, 319, 810-813; v) B. Huang, S. A. Jones, B. Brandenburg, X. Zhuang, Nat. Methods 2008, 5, 1047-1052.

[3] J. Fölling, M. Bossi, H. Bock, R. Medda, C. A. Wurm, B. Hein, S. Jakobs, C. Eggeling, S. W. Hell, Nat. Methods 2008, 5, 943-945.

[4] See the Supporting Information for detailed characteristics of commercially available dyes whose fluorescence can be exited with the $\mathrm{He}-\mathrm{Ne}$ red laser $(633 \mathrm{~nm})$ or the $635 \mathrm{~nm}$ spectral line of red diode lasers.

[5] a) J. E. Berlier, J. Histochem. Cytochem. 2003, 51, 1699-1712; b) B. Agnew, K. R. Gee, T. G. Nyberg (Invitrogen), US 0249014, 2007.

[6] In carbopyronines, as compared to rhodamines, the oxygen atom at position 10 of the xanthene fragment is "replaced" with a geminal dimethyl group $\left[\mathrm{C}\left(\mathrm{CH}_{3}\right)_{2}\right]$. This produces a large bathochromic shift in the absorption and emission bands (ca. $50 \mathrm{~nm}$ in comparison with the corresponding rhodamines): a) J. Arden-Jacob, J. Frantzeskos, A Zilles (K.-H. Drexhage), WO 64986, 2000; b) A. Zilles, J. ArdenJacob, K.-H. Drexhage, N. U. Kemnitzer, M. Hammers-Schneider (Atto-tec GmbH), WO 003086, 2005.

[7] V. Westphal, S. O. Rizzoli, M. A. Lauterbach, D. Kamin, R. Jahn, S. W. Hell, Science 2008, 320, 246-249.

[8] For recent examples, see: a) K. Peneva, G. Mihov, F. Nolde, S. Rocha, J. Hotta, K. Braeckmans, J. Hofkens, H. Uji-i, A. Hermann, K. Müllen, Angew. Chem. 2008, 120, 3420-3423; Angew. Chem. Int. Ed. 2008, 47, 3372-3375; b) C. Jung, B. K. Müller, D. C. Lamb, F. Nolde, K. Müllen, C. Bräuchle, J. Am. Chem. Soc. 2006, 128, $5283-$ 5291; c) S. L. Niu, G. Ulrich, R. Ziessel, A. Kiss, P.-Y. Renard, A Romieu, Org. Lett. 2009, 11, 2049-2052; d) Z. Lu, N. Liu, S. J. Lord, S. D. Bunge, W. E. Moerner, R. J. Twieg, Chem. Mater. 2009, 21, 797-810; e) M. Sauer, K.-T. Han, R. Müller, S. Nord, A. Schulz, S Seeger, J. Wolfrum, J. Arden-Jacob, G. Deltau, N. J. Marx, C. Zander, K.-H. Drexhage, J. Fluoresc. 1995, 5, 247-261.

[9] a) L. G. Lee, R. J. Graham, W. E. Werner, E. Swartzman, L. Lu, (Apptera Corp., USA), US 6372907, 2002. b) Similar spectral values (624 and $644 \mathrm{~nm}$ for absorption and emission, respectively) have been recorded in ethanol for ethyl esters of the tetrachlororhodamines. ${ }^{[6 b]}$

[10] T. Karstens, K. Kobs, J. Phys. Chem. 1980, 84, 1871-1872.

[11] T. Nguyen, M. B. Francis, Org. Lett. 2003, 5, 3245-3248.

[12] M. Bossi, V. N. Belov, S. Polyakova, S. W. Hell, Angew. Chem. 2006, 118, 7623-7627; Angew. Chem. Int. Ed. 2006, 45, 7462-7465.

[13] For an example, see: F. Mao, W.-Y. Leung, R. P. Haugland (Molecular Probes Inc., USA), US 6130101, 2000.

[14] L. A. Robinson, M. E. Theoclitou, Tetrahedron Lett. 2002, 43, $3907-$ 3910 (see also: C. S. Yi, S. Y. Yun, J. Am. Chem. Soc. 2005, 127, 17000-17006)

[15] R. Hermann, H.-P. Josel, K.-H. Drexhage, J. Arden-Jacob (Boehringer Mannheim GmbH), US 57550409, 1998.

[16] a) W. Siebrand, D. F. Williams, J. Chem. Phys. 1968, 49, 1860-1871; b) C. Dosche, M. U. Kumke, H.-G. Lömannsröben, F. Ariese, A. N. Bader, C. Gooijer, O. S. Milanic, M. Iwamoto, K. P. C. Vollhardt, R Puchta, N. J. R. van Eikema Hommes, Phys. Chem. Chem. Phys. 2004, 6, 5476-5483; c) M. J. Frampton, G. Accorsi, N. Armaroli, J. E. Rogers, P. A. Fleitz, K. J. McEwan, H. L. Anderson, Org. Biomol. Chem. 2007, 5, 1056-1061.

[17] a) J. Widengren, U. Mets, R. Rigler, J. Phys. Chem. 1995, 99, 13368 13379; b) C. Eggeling, J. Widengren, L. Brand, J. Schaffer, S. Felekyan, C. Seidel, J. Phys. Chem. A 2006, 110, 2979-2995.

[18] a) S. W. Hell, V. N. Belov, K. Kolmakov, V. Westphal, M. A. Lauterbach, S. Jakobs. C. A. Wurm, C. Eggeling, C. Ringemann (MaxPlanck-Gesellschaft zur Förderung der Wissenschaften e.V.), EP-A 09005886.8, 2009; b) R. Schmidt, C. A. Wurm, A. Punge, A. Egner, S. Jakobs, S. W. Hell, Nano Lett. 2009, 9, 2508-2510.

[19] See the Supporting Information for figures and experimental data.

[20] E. Abbe, Arch. f. Mikroskop. Anat. 1873, 9, 413-420

[21] a) S. Van de Linde, U. Endesfelder, A. Mukherjee, M. Schüttpelz, G. Wiebusch, S. Wolter, M. Heilemann, M. Sauer, Photochem. Photobiol. Sci. 2009, 8, 465-469; b) J. Vogelsang, T. Cordes, C. Forthmann, C. Steinhauer, P. Tinnefeld, Proc. Natl. Acad. Sci. USA 2009 106, 8107-8112; c) M. Heilmann, S. Van de Linde, A. Mukherjee, M. Sauer, Angew. Chem. 2009, 121, 7036-7041; Angew. Chem. Int. Ed. 2009, 48, 6903-6908.

[22] C. Eggeling, C. Ringemann, R. Medda, G. Schwarzmann, K. Sandhoff, S. Polyakova, V. N. Belov, B. Hein, C. von Middendorff, A Schönle, S. W. Hell, Nature 2009, 457, 1159-1163.

Received: August 20, 2009 Published online: November 30, 2009 